\title{
NOTE ON LIMITS OF SIMPLY CONTINUOUS AND CLIQUISH FUNCTIONS
}

\author{
JANINA EWERT \\ Department of Mathematics \\ Pedagogical University \\ Arciszewskiego 22 \\ 76-200 Slupsk, Poland
}

(Received May 18, 1992 and in revised form June 14, 1993)

\begin{abstract}
The main result of this paper is that any function $f$ defined on a perfect Baire space $(X, T)$ with values in a separable metric space $Y$ is cliquish (has the Baire property) iff it is a uniform (pointwise) limit of sequence $\left\{f_{n}: n \geq 1\right\}$ of simply continuous functions. This result is obtained by a change of a topology on $X$ and showing that a function $f:(X, T) \rightarrow Y$ is cliquish (has the Baire property) iff it is of the Baire class 1 (class 2) with respect to the new topology.
\end{abstract}

KEY WORDS AND PHRASES. Simply continuity, cliquishness, function with the Baire property, function of a Baire class $\alpha$.

1991 AMS SUBJECT CLASSIFICATION CODES. 54C08, 26 A21.

Let $(X, T)$ be a topological space and $(Y, d)$ a metric one. A function $f: X \rightarrow Y$ is called:

- simply continuous (with the Baire property) if for each open set $V \subset Y$ the set $f^{-1}(V)$ is the union of an open set and a nowhere dense set [6], (resp. $f^{-1}(V)$ has the Baire property [5]);

- cliquish, if for any point $x \in X, \varepsilon>0$ and for each neighborhood $U$ of $x$ there is a nonempty open set $U_{1} \subset U$ such that $d\left(f\left(x^{\prime}\right), f\left(x^{\prime \prime}\right)\right)<\varepsilon$ for $x^{\prime}, x^{\prime \prime} \in U_{1},[10]$.

For a function $f$ by $C(f)$ we denote the set of all points at which it is continuous. The following results on cliquish functions are known:

(1) If $f$ is cliquish, then $X \backslash C(f)$ is of the first category [7].

(2) If $(X, T)$ is a Baire space and for a function $f: X \rightarrow Y$ the set $X \backslash C(f)$ is of the first category, then $f$ is cliquish [2].

Since for any function $f: X \rightarrow Y$ the set $X \backslash C(f)$ is of the form

$$
X \backslash C(f)=\bigcup\left(f^{-1}(V) \backslash \text { Int } f^{-1}(V)\right),
$$

where the union is taken under all open sets $V \subset Y$ (or all open sets belonging to some base), from (1) we immediately obtain:

(3) Let $X$ be a Baire space and $Y$ a separable metric space. A function $f: X \rightarrow Y$ is cliquish if and only if for each open set $V \subset Y$ the set $f^{-1}(V)$ is the union of an open set and a set of the first category.

Therefore, under assumptions on $X$ and $Y$ as in (3), we have:

$$
\text { simply continuity } \Rightarrow \text { cliquishness } \Rightarrow \text { Baire property }
$$

and it is known that these implications are not reversible. 
LEMMA 1. Let $(X, T)$ be a Baire space and $(Y, d)$ a metric space. If $f: X \rightarrow Y$ is a cliquish function such that $f(X)$ is a countable discrete subspace of $Y$, then $f$ is simply continuous.

PROOF. Let $f(X)=\left\{y_{n}: n \geq 1\right\}$ and let $V_{n}$ be a neighborhood of $y_{n}, n \geq 1$, such that $V_{n} \cap f(X)=\left\{y_{n}\right\}$. So, according to (3) we have $f^{-1}\left(y_{n}\right)=f^{-1}\left(V_{n}\right)=W_{n} \cup H_{n}$, where $W_{n}$ is open, $H_{n}$ is of the first category and $W_{n} \cap H_{n}=\emptyset$; moreoever $\left(W_{n} \cup H_{n}\right) \cap\left(W, \cup H_{\jmath}\right)=\emptyset$ for $n \neq J$ and $\bigcup_{n=1}^{\infty} W_{n} \cup \bigcup_{n=1}^{\infty} H_{n}=X$. It implies $\left(\bigcup_{n=1}^{\infty} W_{n}\right) \cap\left(\bigcup_{n=1}^{\infty} H_{n}\right)=\emptyset$, and since $X$ is a Baire space, the set $\bigcup_{n=1}^{\infty} W_{n}$ is dense. Thus $\bigcup_{n=1}^{\infty} H_{n} \subset \operatorname{Fr}\left(\bigcup_{n=1}^{\infty} W_{n}\right)$. From this it follows that $\bigcup_{n=1}^{\infty} H_{n}$ is a nowhere dense set, which finishes the proof.

A topological space $(X, T)$ is said to be perfect if each open subset of $X$ is an $F_{\sigma}$ set, [1]. We remind that a function $f: X \rightarrow Y$ is called of a Baire class $\alpha$ if for each open set $V \subset Y$ the set $f^{-1}(V)$ is of the additive class $\alpha,[5]$. In [5, p. 388 Th. 3 and p. 390 Th. 6] are proved the following results:

(4) If $X$ is a metric space and $Y$ a separable metric one, then each function $f: X \rightarrow Y$ of a Baire class $\alpha>0$ is a uniform limit of functions $f_{n}, n \geq 1$, of the same class $\alpha$ such that all sets $f_{n}(X)$ are countable discrete subspaces of $Y$.

(5) If $X$ is a metric space and $Y$ a separable metric one, then each function $f: X \rightarrow Y$ of a Baire class $\alpha>1$ is a pointwise limit of functions $f_{n}, n \geq 1$, of a class less than $\alpha$.

Analyzing the proofs of these theorems it can be seen that from properties of a metric space $X$ is needed only that each open set in $X$ is $F_{\sigma}$. Thus [5, pp. 388-390] contains some more general results, namely:

(6) in (4) if suffices to take $X$ perfect;

(7) Let $X$ be a perfect space and $Y$ a separable metric one. Then each function $f: X \rightarrow Y$ of a Baire class $\alpha>1$ is a pointwise limit of functions $f_{n}, n \geq 1$, of a class less than $\alpha$ such that all $f_{n}(X)$ are countable discrete subspaces of $Y$.

So, using (6) and (7) we will prove our main result:

THEOREM 1. Let $(X, T)$ be a perfect Baire space, $(Y, d)$ a separable metric space and let $f: X \rightarrow Y$ be any function. Then:

(a) $f$ is cliquish if and only if it is a uniform limit of a sequence of simply continuous functions;

(b) $f$ has the Baire property if and only if it is a pointwise limit of a sequence of simply continuous functions.

PROOF. According to (2) any simply continuous function is cliquish (so it has the Baire property). By the standard calculus it can be shown that the uniform convergence preserves the cliquishness; therefore a uniform (pointwise) limit of a sequence of simply continuous functions is cliquish (has the Baire property). Now let us put $T^{*}=\{U \backslash H: U \in T, H$ is a nowhere dense set in $X\}$. Then $T^{*}$ is a topology on $X,\left(X, T^{*}\right)$ is a perfect Baire pace ([3], [8]), and nowhere dense sets in $\left(X, T^{*}\right)$ are exactly the same as in $(X, T),([3],[8])$; thus these spaces have the same families of sets with the Baire property. Furthermore, it is easy to verify that under assumptions on $X$ it holds:

(8) a set $A \subset X$ is $F_{\sigma}$ in $\left(X, T^{*}\right)$ if and only if it is of the form $A=U \cup H$, where $U$ is an $F_{\sigma}$ set (open set) and $H$ is of the first category;

(9) a set $A \subset X$ is of the additive (multiplicative) class 2 in $\left(X, T^{*}\right)$ if and only if it has the Baire property. 
Let $f: X \rightarrow Y$ be a cliquish function. According to (3) and (8) the function $f:\left(X, T^{*}\right) \rightarrow Y$ is of the Baire class 1; then applying (4), (6) and Lemma 1 we obtain that $f$ is a uniform limit of a sequence of simply continuous functions. Finally, let us assume the $f$ is a function with the Baire property. Then it follows from (9) that $f:\left(X, T^{*}\right) \rightarrow Y$ is of the Baire class 2. Now using (7) and Lemma 1 we have that $f$ is a pointwise limit of a sequence of simply continuous functions which finishes the proof.

COROLLARY 1. Let $X$ be a perfect Baire space and $Y$ a separable metric one. A function $f: X \rightarrow Y$ has the Baire property if and only if it is a pointwise limit of a sequence of cliquish functions.

In the case $X=Y=R$ the above corollary makes Theorem 2 in [4].

Finally we will consider some form of convergence which is between uniform and pointwise one. Let $X$ be a topological space and $(Y, d)$ a metric one. Following [9], a sequence $\left\{f_{n}: n \geq 1\right\}$ of functions $f_{n}: X \rightarrow Y$ is said to be quasi-uniformly convergent to a function $f$ if for each point $x_{0} \in X$ and $\varepsilon>0$ there exists $n_{0}$ such that for each $n \geq n_{0}$ there exists a neighborhood $U$ of $x_{0}$ with $d\left(f_{n}(x), f(x)\right)<\varepsilon$ for $x \in U$. Then we have:

THEOREM 2. Let $X$ be a perfect Baire space and $Y$ a separable metric one. A function $f: X \rightarrow Y$ is cliquish if and only if it is a quasi-uniform limit of a sequence of simply continuous (cliquish) functions.

PROOF. If $f$ is a cliquish function, then the conclusion is a simple consequence of Theorem 1(a). Conversely, if $f$ is a quasi-uniform limit of a sequence $\left\{f_{n}: n \geq 1\right\}$ of cliquish functions, then according to [9] we have $\bigcap_{n=1}^{\infty} C\left(f_{n}\right) \subset C(f)$. Under assumptions the set $\bigcap_{n=1}^{\infty} C\left(f_{n}\right)$ is dense $G_{\delta}$ so $C(f)$ is too. Thus $X \backslash C(f)$ is of the first category; in virtue of (2) it means that $f$ is cliquish.

For a family $\mathscr{F}$ of functions by $L_{u}(\mathcal{F}), L_{q u}(\mathcal{F})$ and $L_{p}(\mathcal{F})$ we denote the collection of all uniform, quasi-uniform and pointwise limits of sequences taken from $\mathscr{F}$, respectively. Moreover, let $S, \mathscr{G}$ and $\mathscr{B}$ be families of all functions $f: X \rightarrow Y$ which are simply continuous, cliquish or have the Baire property, respectively. Then our results can be presented in the following:

COROLLARY 2. Let $X$ be a perfect Baire space and $Y$ a separable metric one. Then:

$$
L_{u}(S)=L_{u}(96)=96 ; L_{q u}(S)=L_{q u}(96)=96 ; L_{p}(S)=L_{p}(96)=L_{p}(\mathscr{B})=\mathscr{B} .
$$

Taking into account (3) and (8), under assumptions of Theorem 1 we have:

(10) a function $f:(X, T) \rightarrow Y$ is cliquish if and only if $f:\left(X, T^{*}\right) \rightarrow Y$ is of the Baire class 1 .

Using this fact we obtain a new characterization of cliquish functions, namely:

THEOREM 3. Let $(X, T)$ be a perfect Baire space, $(Y, d)$ a separable metric one and $f: X \rightarrow Y$ any function. Then the following conditions are equivalent:

(a) $f$ is cliquish;

(b) for each $T^{*}$-closed of the second category set $M \subset X$ the restriction $f_{/ M}:\left(M, T_{/ M}^{*}\right) \rightarrow Y$ has a continuity point;

(c) for each $T$-closed of the second category set $M \subset X$ the function $f_{/ M}:\left(M, T_{/ M}^{*}\right) \rightarrow Y$ has a continuity point.

PROOF. Let us denote by $D\left(f_{/ M}\right)$ the set of all points at which $f_{/ M}$ is $T^{*} / M^{\text {-discontinuous. }}$ If $f$ is cliquish, then according to (10), the function $f:\left(X, T^{*}\right) \rightarrow Y$ is of the Baire class 1 . So $f_{/ M}:\left(M, T_{/ M}^{*}\right) \rightarrow Y$ is also of the Baire class 1 . It implies that $D\left(f_{/ M}\right)$ is of the first category in $\left(M, T_{/ M}^{*}\right)$; in the consequence it is of the first category in $\left(X, T^{*}\right)$. But $\left(X, T^{*}\right)$ and $(X, T)$ have the same families of the first category sets ([3], [8]) and $M$ is of the second category, thus 
$M \backslash D\left(f_{/ M}\right) \neq \emptyset$, which finishes the proof of $(\mathrm{a}) \Rightarrow(\mathrm{b})$. The implication (b) $\Rightarrow(\mathrm{c})$ is evident, since $T \subset T^{*}$. Now, let (c) be satisfied. We take a point $x_{0} \in X, \varepsilon>0$ and any neighborhood $U$ of $x_{0}$. Then the function $f_{\mid \bar{u}}$ is $T_{\mid \bar{u}}^{*}$-continuous at some point $x_{1} \in \bar{U}$; hence there exist an open set $V$ and a nowhere dense set $H$ with $x_{1} \in V \backslash H$ and $d\left(f(x), f\left(x_{1}\right)\right)<\frac{1}{2} \varepsilon$ for $x \in U \cap(V \backslash H)$. Thus $W=U \cap(V \backslash \bar{H}) \neq \emptyset, W \subset U$ and $d\left(f\left(x^{\prime}\right), f\left(x^{\prime \prime}\right)\right)<\varepsilon$ for $x^{\prime}, x^{\prime \prime} \in W$ which finishes the proof.

\section{REFERENCES}

1. ENGELKING, R., General Topology, Warszawa, 1977.

2. FUDALI, T.A., On cliquish functions on product spaces, Math. Slovaca 33 (1983), 53-58.

3. HASHIMOTO, H., On the *-topology and its application, Fund. Math. 91 (1976), 5-10.

4. GRANDE, Z., Sur la quasi-continuité et la quasi-continuité approximative, Fund. Math. 129 (1988), 167-172.

5. KURATOWSKI, K., Topology, vol. I, Warszawa, 1966.

6. NEUBRUNNOVA, A., On transfinite sequences of certain types of functions, Acta Fac. Rer. Natur. Unvv. Comenianae Math. 30 (1975), 121-126.

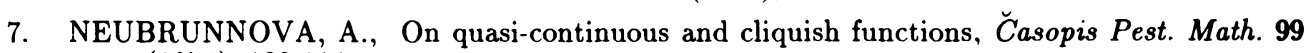
(1974), 109-114.

8. NJÅSTAD, O., On some classes of nearly open sets, Pac. J. Math. 15 (1965), 961-970.

9. PREDOI, M., Sur la convergence quasi-uniforme, Period. Math. Hungarica 10 (1979), 31-40.

10. ThielmaN, H.P., Types of functions, Amer. Math. Monthly 60 (1953), 156-161. 


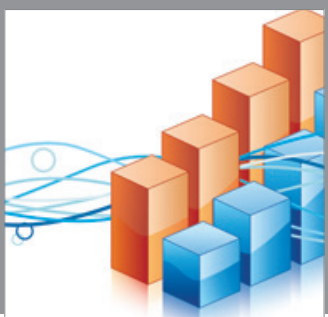

Advances in

Operations Research

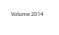

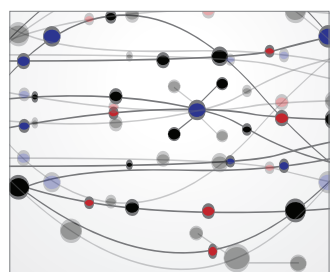

\section{The Scientific} World Journal
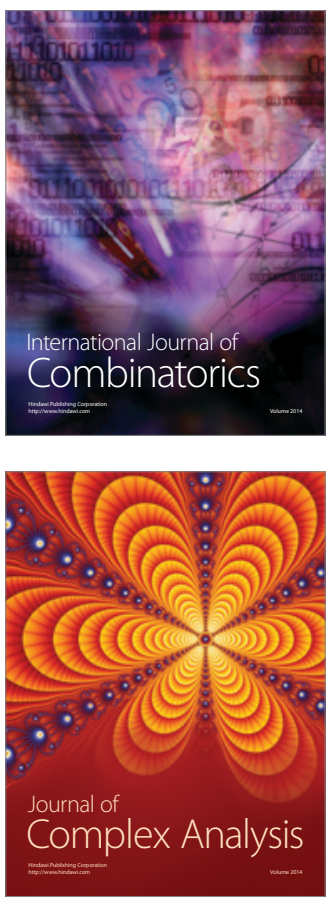

International Journal of

Mathematics and

Mathematical

Sciences
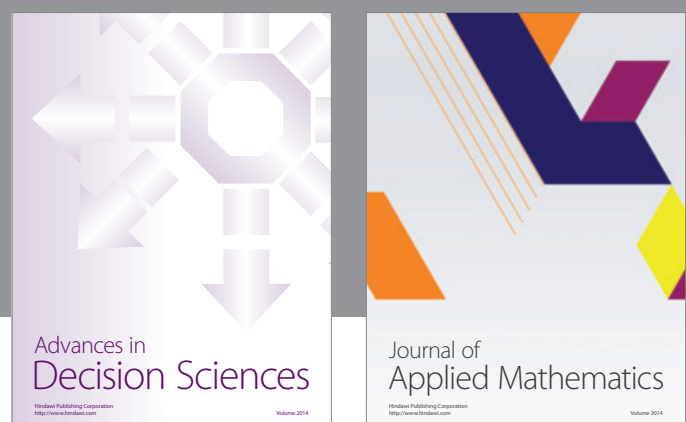

Journal of

Applied Mathematics
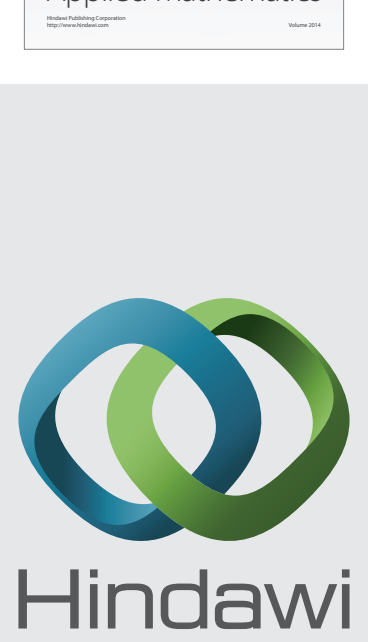

Submit your manuscripts at http://www.hindawi.com
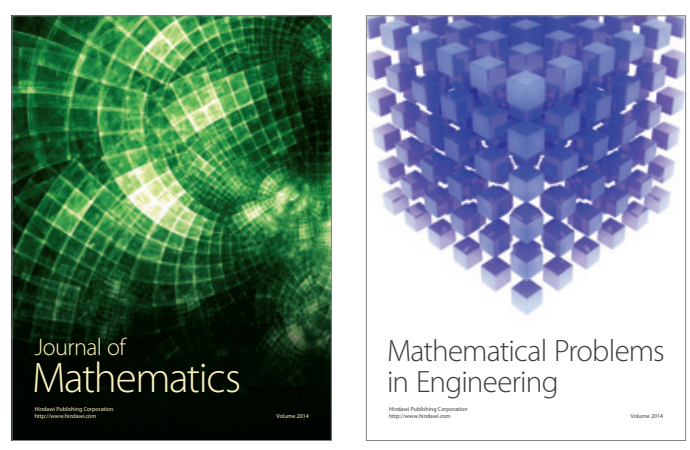

Mathematical Problems in Engineering
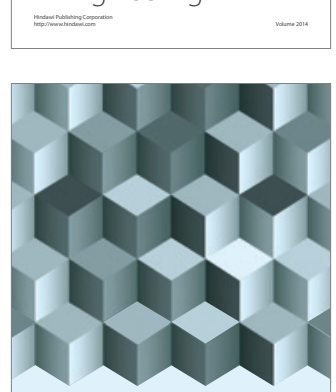

Journal of

Function Spaces
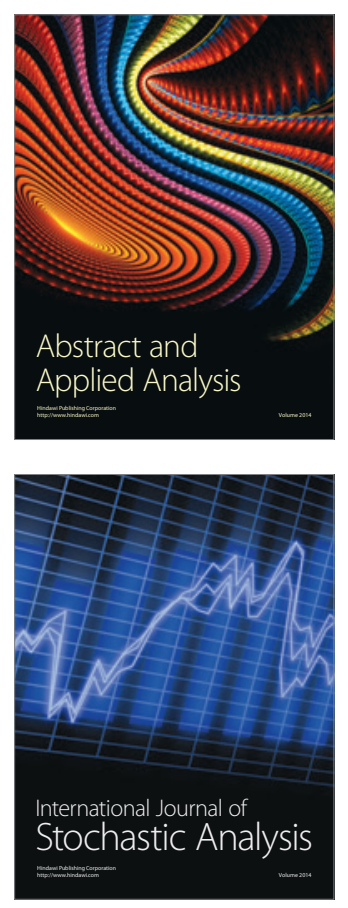

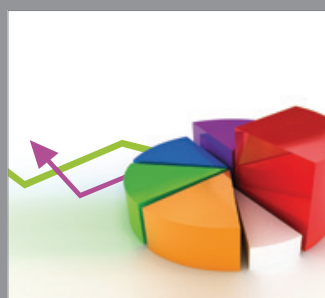

ournal of

Probability and Statistics

Promensencen
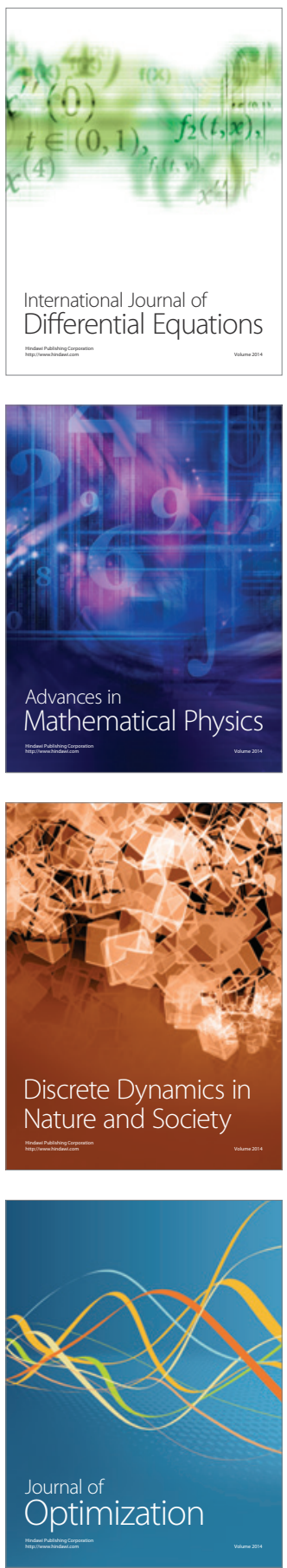